
\title{
(s)
}

\section{Focused Electron Beam Induced Processing Renders at Room Temperature a Bose-Einstein Condensate in Koops-GranMat}

\author{
Hans W.P. Koops \\ HaWilKo GmbH, Ernst Ludwig Strasse 16, 64372 Ober-Ramstadt, Germany
}

\begin{abstract}
Focused Electron Beam Induced Processing allows to generate nanocrystalline materials with metallic conductivity and also nanogranular materials with metal crystals embedded in a fullerene matrix, which shows at room temperature 1000 times better conductivity than superconductors at $40 \mathrm{~K}$ due to a Bose Einstein Condensate. Resistors and field emitters carry $>50 \mathrm{MA} / \mathrm{cm}^{2}$ current density and deliver up to $1 \mathrm{~mA}$ current by field emission, when having an enlarged foot point contact to normal metal like Gold. An explanation for the different characteristics is given. The reason for the generation of the Bose Einstein Condensate is explained, and applications are described.
\end{abstract}

Received on 08-06-2016 Accepted on 13-07-2016 Published on 13-10-2016

Keywords: Field emission, Focused electron beam induced processing, GDSII-layout exposure control, $\mathrm{x},-\mathrm{y},-\mathrm{t},-$-nested loop exposure control, 3-d ebeam printing, Super conductivity, Cooper pairs, Bose Einstein Condensate, Koops-

Pairs, Applications. DOI: http://dx.doi.org/10.6000/2369-3355.2016.03.02.1

\section{INTRODUCTION}

In 1994 a novel material, now called Koops-GranMat ${ }^{\circledR}$ [1], was discovered at FTZ, the German Telekom Research Centre at Darmstadt. This novel material is now understood to have extraordinary characteristics caused by a BoseEinstein Condensate at room temperature, which makes it possible to replace cooled superconducting materials, and offers the chance to revolutionalize many tasks: energy transport and distribution, high frequency electronics above $30 \mathrm{GHz}, \mathrm{THz}$ switching and spectroscopy, materials analysis, photonics and energy harvesting.

The long lasting discussion how this novel material has been generated, but could not be reproduced by other researchers is now understood [2].

\section{ELECTRON BEAM INDUCED DEPOSITION AND ETCHING}

\section{A. Early work}

With the development of early electron microscopes since 1930, which challenged the resolving power of optical

"HaWilKo GmbH, Ernst Ludwig Strasse 16, 64372 Ober-Ramstadt, Germany; Tel: 004961542733; Fax: 00496154631697; E-mail: hans.koops@t-online.de microscopes, the influence of the electron beam in the microscope and its vacuum conditions was found to change the samples. Heide [3] investigated this influence of the sample temperature and the water content in the microscope and explained it by etching with water or deposition of contaminants. Later the destruction of Nitrocellulose films was used by Rau [4] to structure the sample carrier foil with electrons, ions and X-rays and showed the deep etched groves by shadow evaporation in the TEM. These processes were applied to generate transmission gratings for spectroscopy of soft X-Rays from ionized carbon with = $4,4 \mathrm{~nm}$ from flares of the sun [5]. Since field aberrations in electron projection systems for the production of $50 \mathrm{~nm}$ node like transmission line gratings were to be measured [6], organometallic precursors were employed in the sample containing sub cells to record gratings in the image field of the projection systems, and to overlay in double exposures the undistorted centre part of the image field with the edge parts of the image field in a half dose double exposure. The appearing moiré patterns allowed measuring the distortions with sub $\mathrm{nm}$ resolution. Later $80 \mu \mathrm{m}$ diameter quantum dot field arrays could be fabricated with $200 \mathrm{~nm}$ square size using gold precursors in a TEM with Twin lens and a material supply integrated in the sample holder [7]. 


\section{B. Recent Findings}

Triggered by a relocation of an SEM with liquid nitrogen baffle, used to absorb the ultra high vacuum capable diffusion pump oil and it's vapours, we built by electron beam induced deposition a $10 \mu \mathrm{m}$ high carbon needle having a diameter of $200 \mathrm{~nm}$ [8], and also Atomic Force Microscope (AFM) needles on top of the tip of the cantilevers, as well as on etched tungsten needles. Later 3-tip pointed AFM structures were deposited and allowed to probe the deep trench etching in the semiconductor storage devices [9]. When investigating very different organometallic precursors for their structure of the deposited material by using high resolution TEM at MPI Halle and TU Darmstadt, very different characteristics of the deposited material were found [10]. Very fine granular composites based on compounds of carbonyls were discovered, see Figure 1 left. The material crystals percolate and the current conductivity stops at $156 \mathrm{kA} / \mathrm{cm}^{2}$. However, with Gold acetyl-acetonates and cyclopentadienyl Pt compounds, a nanogranular crystalline compound material with a Fullerene type matrix between non percolating metal crystals was found; see Figure 1, middle and right. The metal and the carbon form a common Fermi level at about $5 \mathrm{eV}$, charging the metal negative, and the carbon compound positive. The Fullerene embedding matrix allows that surface orbital states around the platinum or gold nanocrystals can be formed and occupied with electrons from the due to Maxwell temperature distribution at room temperature occupied conduction band levels of the connector material. Those can enter by hopping into the excitonic surface orbitals around the metal crystals, and immediately form Bosons with the holes from the carbonaceous matrix.

The $\mathrm{Pt} / \mathrm{C}$ and $\mathrm{Au} / \mathrm{C}$ materials delivered in field emission outstanding currents $>1 \mathrm{~mA}$, and also emission current densities up to $3 \mathrm{GA} / \mathrm{cm}^{2}$, without any sign of melting at the tip. All three materials were deposited using dwell times > msec or continuous deposition at one spot with a beam current $<2 \mathrm{nA}$ and up to $20 \mathrm{keV}$ accelleration voltage from a cold 310 oriented field emitter. The energy density at the sample ranged up to $60 \mathrm{MW} / \mathrm{cm}^{2}$, which corresponds to 0.3 of the energy density at the surface of the sun, or is aequivalent to the radiation damage dose at a distance of $100 \mathrm{~m}$ from the atomic bomb. The molecules are totally stripped from ligands and the metal atoms could crystallize at $380^{\circ} \mathrm{C}$ to $400^{\circ} \mathrm{C}$ to nanocrystals of $2 \mathrm{~nm}$ diameter (Pt) or $4 \mathrm{~nm}$ diameter (Au), until the fullerenes started to crystallize at $150^{\circ} \mathrm{C}$ and stopped the metal crystal growth, respectively initiated the growth of the neighboring crystal. This process led to a homogenuous compound material of $\mathrm{Pt} / \mathrm{C}$ or $\mathrm{Au} / \mathrm{C}$ [12].

\section{MECHANISM OF CONDUCTION IN NANOGRANULAR MATERIAL AND KOOPS-GRANMAT ${ }^{\circledR}$}

Nanogranular material deposited from metal-carbonyl precursors shows in the high resolution TEM image very small, crystals, which all percolate and are composed from conductors of very similar conductivity. Therefore this material behaves like a resistor with conductivities in the range of a few $\mu \mathrm{Ohm} \mathrm{cm}$. The Koops-GranMat ${ }^{\circledR}$ materials however have non percolating large metal crystals of up to 1000 atoms, which are pure metal, and are embedded in a fullerene or nano-diamond carbon matrix. The average thickness of the C-matrix is $1 \mathrm{~nm}$. The metal crystals are surrounded by surface orbitals around the crystal, which can also be excitonic orbitals. Due to the large orbital diameter and the low binding energy the electrons which are occupying such orbitals have according to the Bohr Theory a wavelength of $2 \mathrm{~nm}$. Since the crystals are $2 \mathrm{~nm}$ in diameter, the energy difference between the excitonic orbitals in the case of Pt/C was $125 \mathrm{meV}$, and for Au/C $65 \mathrm{meV}$, see Figure 2.

According to Bose it is a precondition that a condensate energy level of one energy exists throughout all the material. This is the case, since surface exciton orbitals with $n>5$ overlap with the levels $n>5$ of the neighbouring metal crystal. This allows 2 Fermions, electron and hole, to combine to 1 Boson. Excitons, electron-hole pairs, were predicted by Boer et al. in 1961 to condense at low temperature and high density. In superconducting materials Cooper-Pairs have charge 2, and spin 0. In Koops-GranMat ${ }^{\circledR}$ having Koops-Pairs one electron and one hole have charge 0 , and 2 parallel spins: have spin 1. The Condensate level can then be populated by very many Bosons, which was observed in our experiments. The Bosons are all coherent. This results in
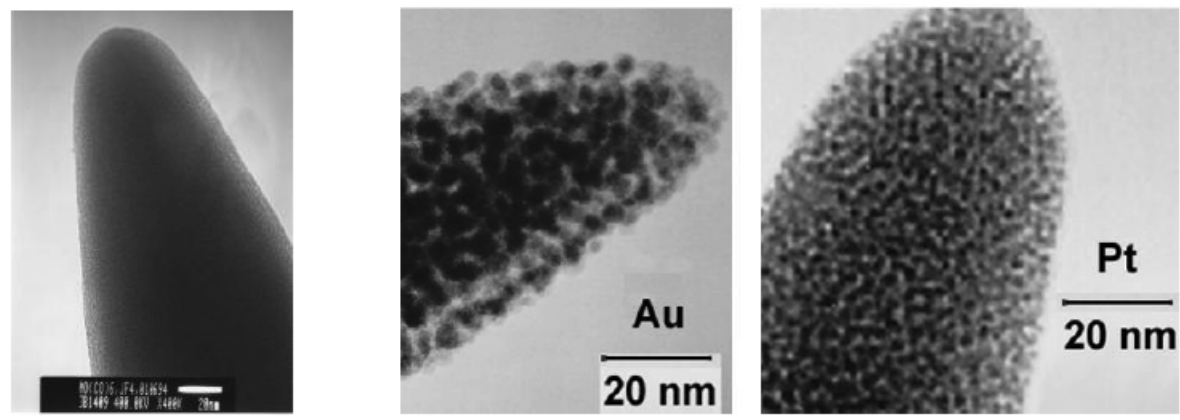

Figure 1: Left carbonylic deposit with percolating nanocrystals from $\mathrm{Mo}(\mathrm{CO}) 6$. Middle and right: Non-percolating crystals from Di-methyl-Auacetyl-acetonate, middle, and from Pt-cyclopentadienyl-trimethyl, right, are embedded in a Fullerene matrix [11]. Au crystals have $4 \mathrm{~nm}$ diameter, Pt crystal $2 \mathrm{~nm}$ diameter. 


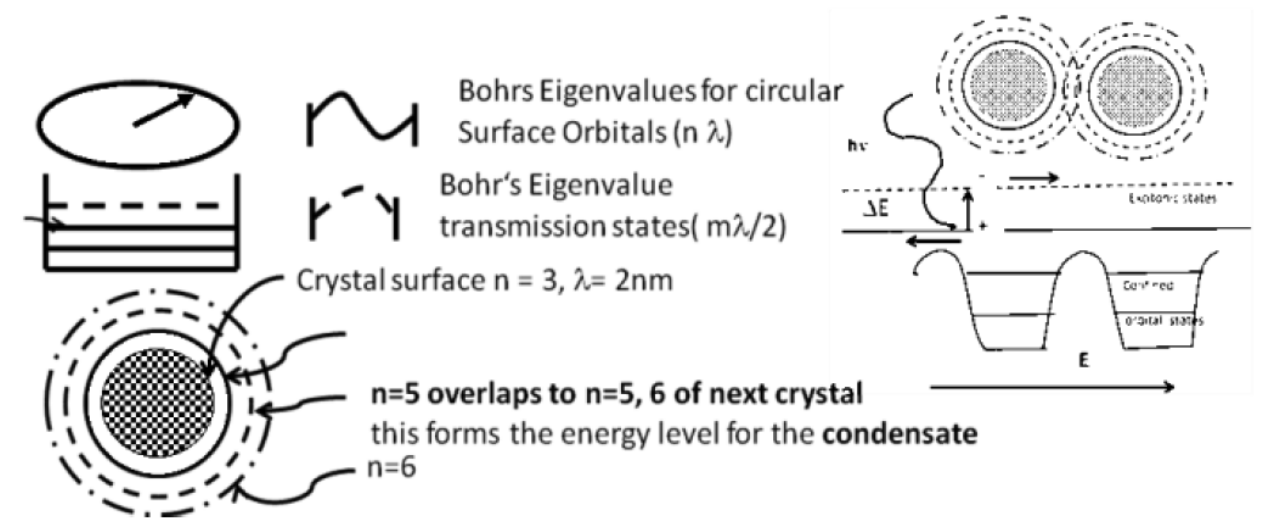

Figure 2: Excitonic orbitals around the nanocrystals overlap with such of the neighbouring crystals and form a Bose-Einstein Condensate [13], which extends through all the deposit.

coherent electron emission from field emitter tips build with Koops-Pairs. This was observed imaging in the far field three Young's interference patterns from one Pt/C emitter tip [14].

Using a theory from L. Butov and M. Remeika, the temperature for the forming of the Bose Einstein Condensate could be calculated, see Figure 3 [15].

\section{CONDUCTION IN KOOPS-GRANMAT ® IS MADE POSSIBLE BY THE MAXWELL STATISTICS DISTRIBUTING ELECTRONS IN THE CONDUCTION BAND OF GOLD AS A CONTACT MATERIAL}

The activation energy for supplying of electrons from normal metal conduction band levels to the Koops-GranMat ${ }^{\circledR}$ was measured with a Poole-Frenckel setup to be for Pt/C 125 $\mathrm{meV}$, and for Au/C $65 \mathrm{meV}$ [16]. Theoretically the excitonic surface orbitals show these energies as a band gap for the excitonic surface orbitals around the crystals [17]. This result corresponds to the measured data.

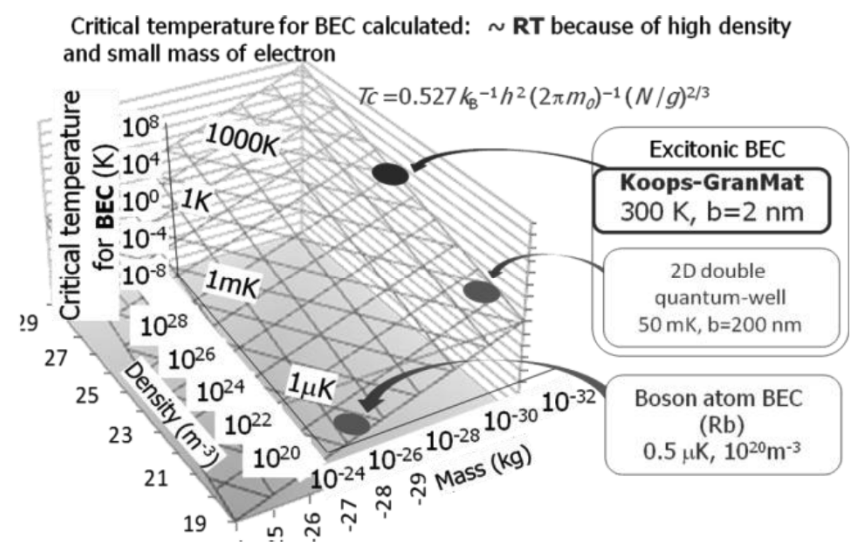

Figure 3: Calculated Bose-Einstein condensate temperature TC $\mathrm{BEC}$ as a function of the mass and the density of bosons for Rubidium $(0.5 \mu \mathrm{K})$, for the excitonic BEC in 2D-double Quantum wells $(50 \mathrm{mK})$, and for excitonic BEC in Koops-GranMat (300 K).

\section{A: Energy states in Koops-GranMat ${ }^{\circledR}$}

Table 1 shows the result of the use of Bohr's atom model to estimate the energy and diameters of the excitonic orbital states. Bohr radius $r=\varepsilon n^{2} h^{2} /\left(\pi m_{\text {eff }} e^{2}\right)$; Energy level: $E=m_{\text {eff }} e^{4} /\left(8 e^{2} n^{2} h^{2}\right)$

\section{B: Current Conduction from the Connecting Metal to the Koops-GranMat ${ }^{\circledR}$}

In Figure 4 is the energy level situation depicted. From the 3D Gold metal contact at the border to Koops-GranMat ${ }^{\circledR}$ energy levels electrons hop into the excitonic levels around the metal crystals. Since the contact is normally at room temperature, the energy levels of the metal conduction band are partially occupied, even at higher voltage, due to the in this material acting Maxwell temperature law for level occupation. Since in the Koops-GranMat ${ }^{\circledR}$ the overlapping energy levels of the condensate are about $250 \mathrm{meV}$ above the room temperature, such levels would normally not be occupied. But due to the energy distribution due to the Maxwell statistics in the connecting gold material, several electrons have already the energy to tunnel directly into the excitonic energy level of the BEC condensate. Now a current can flow to the KoopsGranMat $^{\circledR}$, and form Bosons and result in current flow with zero resistance by coherent Bosons. Since the Koops-pair has a compensated charge but a strong dipole moment, the Boson can be moved only by an inhomogeneous electric field, or an electric field with a gradient.

\section{GIANT CURRENT CARRYING CAPABILITY IS OBSERVED IN KOOPS-GRANMAT ${ }^{\circledR}$}

When measuring the field emission and the conductivity in deposited lines between gold contacts not really understandable values were measured. Field-emission measurements using $A u / C$ rendered a maximum emission current of almost $1 \mathrm{~mA}$ at $22 \mathrm{~V}$ extraction voltage [18], and from $\mathrm{Pt} / \mathrm{C}$ of $1.3 \mathrm{~mA}$ at 70V [19]. Typical Mo emitters stop emission at $10 \mu \mathrm{A}$ but at an extraction voltage $>100 \mathrm{~V}$ [20]. In comparison to the current density values known for superconductors a similar mechanism was to be assumed [21].

In Table 2 a comparison is given for current densities in superconductors, see last line, and also for Koops-Gran-Mat ${ }^{\circledR}$ 
Table 1: Using Bohr's Model: The Predicted E between Confined and Overlapping Orbit Agree with Observed Ea for Pt and Au

\begin{tabular}{|c|c|c|c|c|}
\hline Material & $\begin{array}{c}\text { Exciton diameter } \\
\mathbf{r}(\mathbf{n m})\end{array}$ & Exciton number & $\begin{array}{c}\text { Energy difference } \\
\mathbf{E}(\mathbf{m e V})\end{array}$ & $\begin{array}{c}\text { Observed } \\
\text { EA (meV) }\end{array}$ \\
\hline \hline $\mathrm{Pt}$ & 3 & $5-6$ & 166 & 125 \\
\hline $\mathrm{Au}$ & 7 & $6-7$ & 65 & 65 \\
\hline
\end{tabular}

which surpasses the values for superconductors by orders of magnitudes.

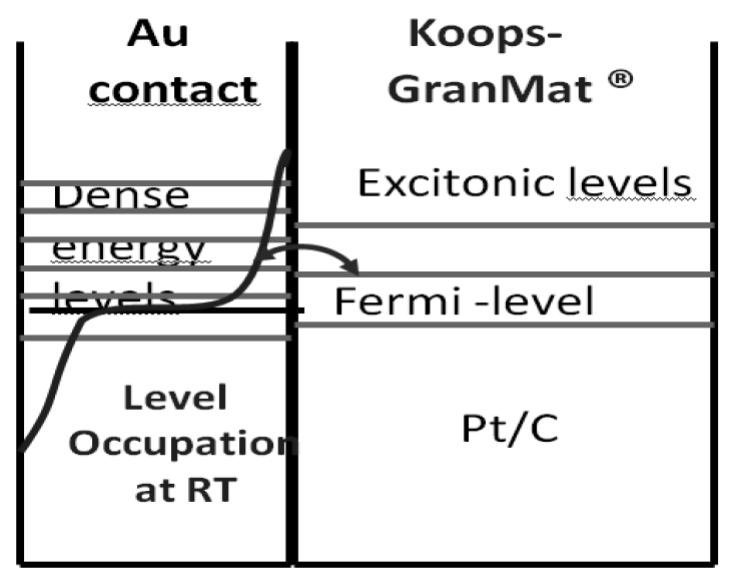

Figure 4: Energy levels and electron occupation at room temperature at the contact of metal and the Koops-GranMat ${ }^{\circledR}$. The Maxwell statistic lifts the electrons in the conduction band of the contact material gold up to the common Fermi-level and allows a direct tunneling into the Koops-GranMat ${ }^{\circledR}$.

Table 2: Comparison of the Capability to Carry Current Densities for Superconductors and Koops-GranMat ${ }^{\Theta}$, as Measured at the German Telekom Research Centre $[8,9,15,22,18]$ and by other Researchers at NaWoTec [22] and at University Maryland [23]

\begin{tabular}{|c|c|c|}
\hline Review FEBIP & $\mathrm{MA} / \mathrm{cm}^{2}$ & {$[8]$} \\
\hline Au/C Field emitter- RT & 2 , at tip 1000 & {$[9]$} \\
\hline Pt/C Field emitter- RT & 2 & {$[15]$} \\
\hline Pt/C Wire arch- RT & 15 & {$[22,23]$} \\
\hline Pt/C FE Emitter- RT & 10 & {$[18]$} \\
\hline Pt/C Wire - RT & 100 & {$[21]$} \\
\hline HTC Superconductors Ti/MgB2-40 K & $<1$ & {$[20]$} \\
\hline
\end{tabular}

\section{A: Superconductivity and Bose Einstein Condensate BEC at Room Temperature}

Superconductivity, as it was explained by Bardeen, Cooper, Shrieffer, did not teach the truth [24]. Just recently a group of scientists from Max Planck foundation, CEA-CNRS, University Dresden, Munich, and Goettingen, published a statement "Our observations point to a surprisingly simple theoretical description of the spin dynamics in the iron arsenides and provide a solid foundation for models of magnetically mediated superconductivity" in 20.12.2009 [25]. Also B. Steele stated in 2014, "Magnetism glues Cooper-
Pairs" [26]. This statement was based on the work of J. C. S. Davis, Cornell University and S. Avici et al. May 2014 [27], who published measurements which confirm that the Cooper pair is stabilized by a balance of charges and spins. The same statement was given in a movie by CNRS in Youtube showing how the Cooper pairs form and then finally all Cooper pairs become Bosons, and form a coherent wave in the superconducting material [28]. The Bosons in this case are formed from 2 electrons, having antiparallel spin. This spin configuration stabilizes the Boson at a diameter of 600 $\mathrm{nm}$ !, see Figure 5, left. Having encountered in our by FEBIP produced Koops-GranMat ${ }^{\circledR}$ the capability to transport current densities at room temperature which are > 1000 times the value of HTC-Superconductors, I defined "Koops-Pairs" as Bosons, formed by an electron and a hole but having parallel spin, see Figure 5, right. In contradiction to this finding, early work of Böer and coworkers in University of New York, USA states, that excitonic electron and hole pairs can exist only at very low temperatures [29].

In the Pt/C- nanogranular matrix compound, as in the $\mathrm{Au} / \mathrm{C}$ compound generated by high dose FEBIP, two phases of different work function are touching ( $\mathrm{Pt}: 5,3 \mathrm{eV}, \mathrm{C}$ : 4,8 eV, or Au: $5,0 \mathrm{eV}$ and $\mathrm{C}: 4,8 \mathrm{eV}$ ). Since the metal crystals are embedded by a fulleren type matrix, the metals attract the electron from the $\mathrm{C}$ and form a common Fermi level. Due to this situation, the metals become negative, and the C-matrix becomes positive. Due to Bohrs atom theory and the Heisenberg equation for energy Eigenstates it is possible that above the common Fermi level unoccupied energy levels exist, which can be occupied by electrons, which are delivered from the conduction band of the gold contact. The Bohr- theory gives for the surface orbitals around the metal crystals energy levels with an energy separation of $125 \mathrm{meV}$ for $\mathrm{Pt}$, and $65 \mathrm{meV}$ for the gold crystals. These excitonic surface orbitals, also follow the Bohrs atom theory, which means, that only energies exist, which allow full wavelength in the perimenter. The excitonic surface orbital which has a perimeter of 5 and higher, however overlap to the ones from the neighbouring crystal. Therefore in the KoopsGranMat $\AA$ a Bose Einstein Condensate can form, and paired Fermions can transit to Bosons. These Bosons are formed from a surplus of electrons and also a surplus of holes in the C-matrix, and these Bosons can occupy this energy level with very many Bosons in parallel, which also become coherent, like in a Laser. This happens at room temperature.

Superconducting material must be cooled, since the material is built as macroscopic crystals in the chemical composition, and such large crystal areas can support high energy 

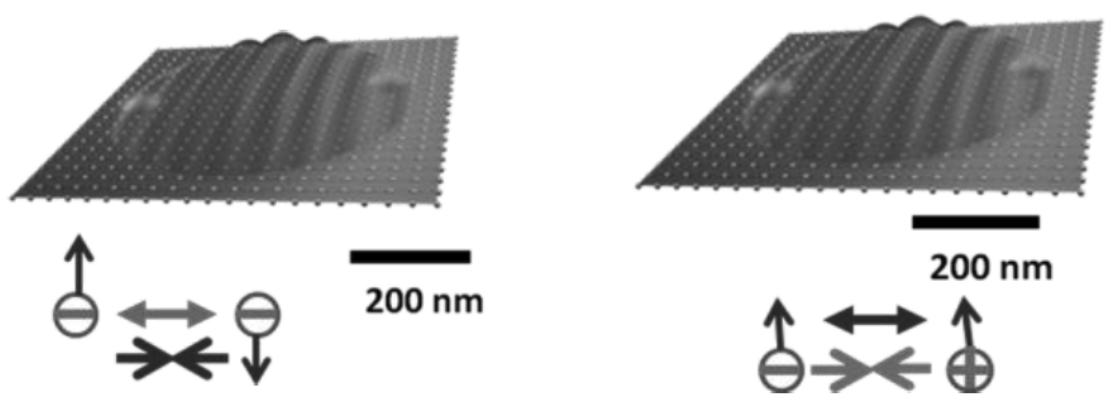

Figure 5: Left: Cooper Pair, 2 charges repell each other, but antiparallel spins contract the charges and balance the Boson. Right: Koops-Pair, electron ( - ) and hole (+) attract each other, but the parallel spins repell each other and stabilize the Boson to a diameter of $600 \mathrm{~nm}$. The only difference between Cooper-pairs and Koops-pairs is in the signs of the charges or of the magnetic force. There is no change in the law for the forces, but the signs are changed.

phonons, which disturb the balancing forces. This makes it, that only below a jump temperature, the Bosons can be formed and the resistivity becomes zero.

Koops-Pairs are generated due to the difference of the epitaxial growth temperature of metal (ca. 380 to $400{ }^{\circ} \mathrm{C}$ ) and carbon or Fullerenes (at $150^{\circ} \mathrm{C}$ ). The growing matter is frozen in to a nanogranular metal crystals structure which is embedded in an at lower condensation temperature formed C-Fullerene matrix. Since this formation is generated during the cooling of the nanogranular matrix compound material according to laws of "mother nature" during the deposition process, all the material has the same characteristics, no matter how long the wire is built.

According to Geller [30], a $5 \mathrm{~nm}$ diameter silicon ball, which is positioned on a Si metal layer can adopt only $10^{-4}$ of the heat in the silicon wafer. This corresponds to $2 \mathrm{meV}$ or $23 \mathrm{~K}$. In our case, however, the metal crystal and the substrate are both made from the same metal, but not percolating, but insulated with respect to each other by a Fullerene matrix. Therefore much less than $10^{-4}$ of the substrate temperature can be adopted. That makes the nanogranular matrix material to be "super cool", and this characteristic allows the Bose -Einstein Condensate to be built even at room temperature of the ambient material. As experiments showed is the contact resistance quite high, up to $\mathrm{MOhm}$ with contact areas of $50 \times 50 \mathrm{~nm}^{2}$. However enlarging the contact area from Koops-GranMat ${ }^{\circledR}$ to the metal reduces the contact resistance, and needs to be designed accordingly to the amount of current which shall be transported from the contact material e.g. Gold ( $250 \mathrm{kA} / \mathrm{cm}^{2}$ ) to the Koops-GranMat ${ }^{\circledR}$. For example, a current of $10 \mathrm{~A}$ requires a contact area of $66 \mu \mathrm{m}$ diameter.

In earlier experiments miniaturized Field-Electron sources delivered electrons at voltages below $100 \mathrm{~V}$ and up to $1 \mathrm{~mA}$ current. This is of great importance for electronics, high speed switching amplifiers, high power sources for IR, THz (up to $6 \mathrm{THz}$ and $1 \mathrm{~W}$ ) and X-Rays, switchable sources for Brachy-therapy, high current switching, high resolution detectors and sensors for IR, Vis, UV and EUV, detectors for $\mathrm{X}$-ray tomography and X-ray phase contrast imaging.
The construction of self reproducing miniaturized optics for massive parallel particle optics [31] and for the application of scanning- electron- and -ion-microscopes for the semiconductor industry becomes possible. With such specially developed adapted multiple beam production machines many novel products can be produced in an economic way, like large area sheets for catalysators, solar cells, long power distributing cables, low voltage high current switches, field-emitter lamps with $65 \%$ efficiency!!, field emitter displays, and many more optical and electronic devices. See the attached patents [32-35].

\section{CONCLUSIONS}

The applications of Koops-GranMat ${ }^{\circledR}$ can revolutionalize the electronic technology in a dramatic way, with the capability of $>100$ times of what super-conducting materials can do, but with the advantage to operate at room temperature and having no need for expensive cooling systems.

\section{ACKNOWLEDGEMENTS}

The author appreciates the cooperation and advice of Dr. Hiroshi Fukuda, Hitachi High Technologies Tokyo, Japan and his theoretical support. He is grateful to Dr. J. Sellmair, Sellmair Nano-electrotechnics GmbH, DE, Prof. T. Gotszalk, from TU Wroclaw PL, and Prof. Ivo Rangelow from TU Ilmenau, DE, and to the RAITH GmbH, DE, for experimental support. He thanks Prof. H. L. Hartnagel, IMP TU Darmstadt, $\mathrm{DE}$ for metrology support and for discussions on $\mathrm{THz}$ radiation applications, also $\mathrm{Dr}$. U. Koops, Ober-Ramstadt, $\mathrm{DE}$, his son, for chemistry discussions, and also $\mathrm{Dr}$. H. Ito, Hitachi High Technologies, Japan, for supports.

\section{REFERENCES}

[1] Koops-GranMat $\circledast$ Name protection recorded in the Register of Community Trademarks in EU, 02.10. 2014, No 012719217 OHIMOffice for Harmonization in the Internal Market.

[2] Huth M, Porrati F, Schwalb C, Winhold M, Sachser R, Dukic M Adams J. Beilstein Journal of Nanotechnology 2012; 3(1): 597-619. http://dx.doi.org/10.3762/bjnano.3.70

[3] Heide G. Z Angewandte Physik 1963; 15: 116.

[4] Rao NV. Z Angewandte Physik 1959; 129: 483. 
[5] Bräuninger $\mathrm{H}$, Einighammer $\mathrm{HJ}$, Feitzinger JV, Fink $\mathrm{HH}$, Höhn $\mathrm{DH}$, Koops H, Krämer G, Mayer G, Möllenstedt G, Mozer M. EUV- and Soft X-Ray Images of the Sun on March 11, 1971. Solar Physics 1971; 20: 81-84. http://dx.doi.org/10.1007/BF00146098

[6] Koops $\mathrm{H}$. Elektronenoptische Herstellung von Transmissionsgittern mit $100 \mathrm{~nm}$ Gitterkonstante für weiche Röntgenstrahlung. Dissertation Tübingen 1971.

[7] Rüb M, Koops HWP, Tschudi T. Electron-beam induced deposition in a reducing image projector. Microelectronic Engineering 1989; 9: 251254. http://dx.doi.org/10.1016/0167-9317(89)90059-2

[8] Hübner B, Koops HWP, Pagnia H, Sotnik N, Urban J, Weber M. Tips for Scanning Tunneling Microscopy Produced by Electron-beam Induced Deposition. Ultramicroscopy 1992; 42-44: 1519-1525. http://dx.doi.org/10.1016/0304-3991(92)90476-Z

[9] Koops HWP, Kretz J, Rudolph M, Weber M, Dahm G, Lee KL. Caractérisation and application of materials grown by electron beam induced deposition. Jpn J Appl Phys 1994; 33: Part. 1 No. 12B: 70997107.

[10] Kretz J, Rudolf M. Diploma thesis works TU Darmstadt Institute of Applied Physics 1994.

[11] Investigating a $\mathrm{Pt} / \mathrm{C}$ deposition sample with Micro-Raman Spectroscopy revealed a Fullerene peak in the spectrum, private communication by Prof. Ion Tiginianu, Academy of Sciences, Chisinau, Moldavia, at his visit at University Freiberg, DE 1998.

[12] Koops HWP, Kretz J, Rudolph M, Weber M. Constructive 3dimensional Lithography with Electron Beam Induced Deposition for Quantum Effect Devices. J Vac Sci Technol B 1993; 11(6): 23862389.

http://dx.doi.org/10.1116/1.586991

[13] Bose SN, Einstein A. 1925 Leiden University Einstein Archive. Lorentz.leidenuniv.nl. 27 October 1920. Retrieved 23 March 2011.

[14] Murakami K, Wakaya F, Takai M. J Vac Sci Technol B 2007; 25(4): 1310-1314 http://dx.doi.org/10.1116/1.2756550

[15] Remeika M. et al. Two-dimensional electrostatic lattices for indirect excitations. Appl Phys Lett 2012; 100: 061103. http://dx.doi.org/10.1063/1.3682302

[16] Koops HWP, Kaya A, Weber M. J Vac Sci Technol B 1995; 13(6): 2400-2403. http://dx.doi.org/10.1116/1.588008

[17] Koops H, Fukuda H. Giant current density via indirect exciton orbit overlapping in polarized nanogranular materials. J Vac Sci Technol B 2015; 33: 02B108.

[18] Koops HWP, Rudolph M, Kretz J, Weber M. Nanolithography in 3 Dimensions with Electron Beam Induced Deposition. NATO ASI
Series E: Applied Sciences Vol. 264, (Gentili M, et al. Eds.) Nanolithography: A Borderland between STM, EB, IB, and X-ray Lithographies. 1994; 87-93. Kluwer Academic Publishers.

[19] Floreani F, Koops HW, Elsäßer W. Concept of a miniaturised freeelectron laser with field mission source. Nuclear Instruments and Methods in Physics Research A 2002; 483: 488-492. http://dx.doi.org/10.1016/S0168-9002(02)00367-4

[20] Spindt CA. J Appl Phys 39: 1968; 3504 http://dx.doi.org/10.1063/1.1656810

[21] Canfield PC, Budco S. Spectrum der Wissenschaften Juni 2005; p. 56.

[22] Sellmair J. NaWoTec GmbH Rossdorf, private communication 2005

[23] Edinger K, Rangelow IW, Gotszalk T. A Novel High Resolution Scanning Thermal Probe. J Vac Sci Technol 2001; B19: 2856-2860. http://dx.doi.org/10.1116/1.1420580

[24] Bardeen J, Cooper LN, Schrieffer JR. Microscopic Theory of Superconductivity. Physical Review 1957; 106(1): 162-164. http://dx.doi.org/10.1103/PhysRev.106.162

[25] Inosov DS, et al. Nature Physics 2010; 6: 178 http://dx.doi.org/10.1038/nphys1483

[26] Steele B. Cornell Chronicle July 28, 2014

[27] Davis JCS. Cornell University, S. Avici et al. Nature Comm. 5, Article number: 3845, 22 May 2014.

[28] http://www.supraconductivite.fr/en/index.php\#supra-explicationcooper, Excerpt from http://www.supraconductivite.fr/en/index.php? $\mathrm{p}=$ recherche-\#supra-explication, http://www.cyclopaedia.fr/wiki/Cooper_electron_pair

[29] Blatt JM, Böer KW, Brandt W. Bose Einstein Condensation of Excitons. Phys Pev 1962; 126: 1691. Pub. 1. 6.

[30] Geller MR, Dennis WM, Markel VA, Patton KR, Simon DT, Yang H-S. Theory of electron-phonon dynamics in insulating nanoparticles. Physica B 2002; 316-317: 430-433. http://dx.doi.org/10.1016/S0921-4526(02)00535-5

[31] Koops HWP. Verfahren und Vorrichtung zur Herstellung von Korpuskularstrahlsystemen DE 0000 10302794A1 4.01.2003.

[32] Koops HWP. "Orbitron pump" Patent family with this title containing DE No. 10241 549, 2002

[33] Koops HWP. Device for generating $\mathrm{THz}$ radiation with free electrons. EP. No. 10170 823.8, 2011.

[34] Koops HWP. "Nano Granular material", European Patent Application No. $12183564.9,2012$.

[35] Koops HWP. QUIDART-Quantum Interference Device at Room Temperature. German Patent Application DE 102014019354 A1 2016.06.23

(c) 2016 Hans W.P. Koops; Licensee Lifescience Global.

This is an open access article licensed under the terms of the Creative Commons Attribution Non-Commercial License (http://creativecommons.org/licenses/by-nc/3.0/) which permits unrestricted, non-commercial use, distribution and reproduction in any medium, provided the work is properly cited. 